COLLOQUE DE PHYSIQUE

Colloque C2, supplément au $n^{\circ} 2$, Tome 51, Février 1990

ler Congrès Français d'Acoustique 1990

\title{
AUTOMATIC SELECTION OF PHONOLOGICALLY COMPACT PHRASES
}

\author{
A. FALASCHI \\ La Sapienza University of Rome, INFOCOM Dept, Via Eudossiana 18. \\ I-00184 Roma, Italy
}

\begin{abstract}
Résumé - Il est exposé un method pour sélectionner automatiquemant un ensemble de phrases avec une composition phonologique maximemant uniforme. La nature des événemants pris en compte est aussi bien syntagmatique que pragmatique. Le method de sélection essaye de donner a tous les événemants ammissible la meme fréquence. Applications de les phrases obtenue comme ensemble d'apprentissage pour systèmes de reconnaisance de la parole et comme ensemble d'essai pour systèmes de synthese sont envisage.
\end{abstract}

\begin{abstract}
A method for automatically select a set of phrases with a maximally uniform phonological composition is exposed. The nature of the is both syntagmatic and paradigmatic. The selection method described tries to give equal frequency to all of the allowable events. Applications of the obtained phrases as a training script for speech recognition systems and as a test one for text-to-speech systems intelligibility evaluation are envisaged.
\end{abstract}

\section{1 - INTRODUCTION}

Voice communication with computers employes real speech for the learning process about the acoustical properties of linguistic descriptions of speech. The composition of this acoustic material should be very carefully selected. In fact, statistical speech recognizer performances depend on how well the training data represents all the acoustic-phonetic events that can occur during the recognition phase. A similar exigency arises for text-to-speech synthesis devices intelligibility assessment, which has to be tested over all the relevant stationary and transitional speech events. Moreover, this sample script should be as short as possible in order to reduce both time (either human or machine) and mass storage requirements.

Here it is proposed to accomplish the above exposed requirements by means of the iterative procedure which is described in Sect 3. It individuates a sub-set of phrases among a larger one by choosing, at each iteration, the not yet selected phrase which contains the majority of events poorly represented into the yet selected list. The selection is based on the definition of a phrase score function, which accounts for both syntagmatic and paradigmatic aspects (in the sense which will be clarified in 3.2) of the phonemic composition of each phrase with respect to these of the selected phrases list.

The selection algorithm operates on a structured, phonetic transcription of text files, in which the phonemic symbols are associated to a couple of indexes that specify their functional role inside the syllable structure $/ 1$, and which philosophy will be resumed in Sect. 2. Such a structural analysis starts from a syllabified phonemic transcription, and can be applied to every language for which this kind of processing is available. For this reason, the selection method here exposed and originally developed for Italian can be directly applied to any other language.

Moreover, the proposed selection method is particularly well suited for natural speech modeling, because the resulting script is constituted by meaningful sentences. Finally, the algorithm score definition allows to focalize the selection on events for which a particular good description is needed.

\section{2 - THE STRUCTURED PHONEMIC TRANSCRIPTION}

This module performs the transcription of written texts into a sequence of Structural Phonetic Units (SPU), by adding to each phoneme label two indices related to its functional role when occurring in different syllabic positions. The SPU definition is based on the concept of functional allophones, which discriminates phonemes on the basis of the syllabic morphology of the language. A more detailed description on this topic can be found in /1/, /2/. 


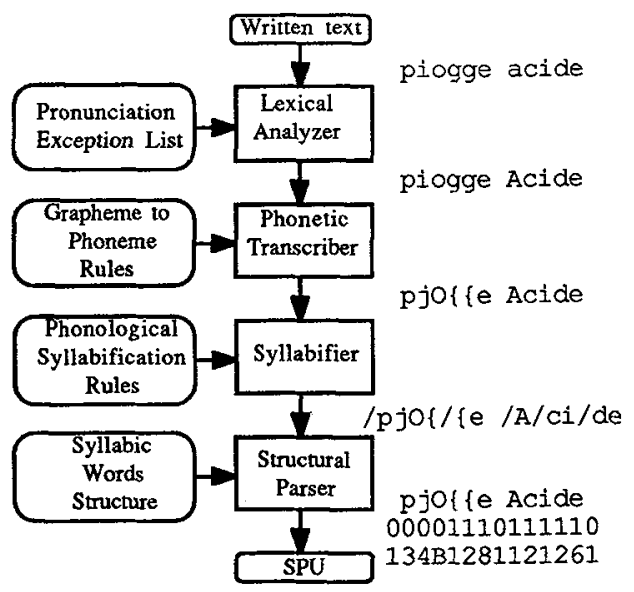

Fig. 1 - SPU Transcription flow-chart

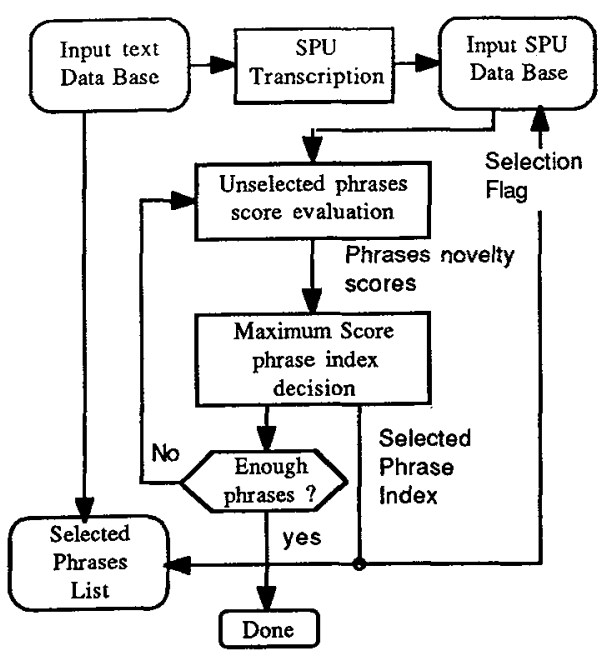

Fig. 2 - Automated phrases selection algorithm structure

The SPU transcription procedure can be summarized as in Fig. 1. The first module deals with non standard pronunciations, as for irregularly stressed or foreign words. The second and third module perform the phonetic transcription and syllabification tasks. The fourth module performs the parsing of the syllabified phonetic string according to stress and syllable automatons, which structure is reported in $/ 1 \%$ The result of the overall process is exemplified at he bottom of Fig. 1, where two structural indexes are attached to each phoneme: the hexadecimal one reports about the phoneme position inside the syllable, and the binary one gives the syllable relative position with respect to the lexical stress.

\section{3 - AUTOMATIC PHRASES SELECTION}

\subsection{Scope and related work}

Reference $/ 3 /$ reports about the design of the TIMIT speech data base, which composition include calibration, phonetically compact and randomly selected sentences. While the calibration set contains mainly dialect-dependent events, the phonetically compact set try to account for all the allowable phonetic contexts in a meaningful, minimum size, sentence list. The randomly selected sentences were chosen by TI from a large database as an integration of the second set, in order to account for phoneme triples in a balanced way, providing the contextual variability which is difficult to obtain by means of an hand-made selection.

Our approach to a phonologically compact data base design is fully automatic. For this reason, it is very close to the one adopted by TI. As a difference, the aim of the automatic procedure here exposed is to obtain a phrases list which phonemic composition results to be maximally uniform under three different points of view: absolute, syntagmatic and paradigmatic ones.

\subsection{The method}

The algorithm operates on the SPU transcription of written texts, and utilizes the very simple but effective procedure exposed in the introduction and summarized by means of Fig. 2. At each iteration of the algorithm, a new phrase is selected from the input DB, on the basis of the score values obtained for the unselected phrases. The result of the selection algorithm is a set of indices, which can be utilized for the retrieval of the selected phrases list in either its orthographic or phonemic form. Let us clarify how the score function is calculated.

\subsection{Score function definition}

The score function must quantify the "complementarity" of the unselected phrases composition with respect to the yet selected list, taking jointly into account the points of view which constitutes the objective of the selection method. In the following, phrase syntagmatic composition will be referred as "sequential", and paradigmatic as "functional".

The score value $S$ for each phrase Pr is computed as a linear combination of Phonemic, Functional and Sequential Score contributions:

$$
\mathrm{S}(\mathrm{Pr})=\lambda_{\mathrm{P}} \mathrm{S}_{\mathrm{P}}(\mathrm{Pr})+\lambda_{\mathrm{F}} \mathrm{S}_{\mathrm{F}}(\mathrm{Pr})+\lambda_{\mathrm{S}} \mathrm{S}_{\mathrm{S}}(\mathrm{Pr})
$$


where the $\lambda_{X}<1$ is the $x$-type score contribution weight coefficient. The constraint $\lambda_{P}+\lambda_{F}+\lambda_{S}=1$ holds. The $S_{X}(\operatorname{Pr})$ value depends on both Pr and the yet selected phrases composition, and it is calculated as:

$$
S_{x}(P r)=\frac{1}{N} \sum_{j=1}^{N} e^{-\alpha_{x} N\left(E_{x}\left(S P U_{j}\right)\right)}
$$

where $\mathrm{N}$ is the number of SPU contained in Pr, the dumping coefficients $\alpha_{\mathrm{x}}$ are any positive number, and $\mathrm{N}\left(\mathrm{E}_{\mathrm{X}}\left(\mathrm{SPU}_{\mathrm{j}}\right)\right)$ is the number of occurrences of the $\mathrm{x}$-type event related to $\mathrm{SPU}_{\mathrm{j}}$, among the yet selected phrases. It is

$$
E_{F}\left(S P U_{j}\right)=S P U_{j} ; \quad E_{P}\left(S P U_{j}\right)=P h_{j} ; \quad E_{S}\left(S P U_{j}\right)=\left\{P h_{j}, P h_{j+1}\right\}
$$

where $\mathrm{Ph}_{\mathrm{j}}$ is the $\mathrm{j}^{\text {th }}$ phoneme of the phrase $\mathrm{Pr}$.

As can be seen from the formulas, the $\mathrm{x}$-type score components is a decreasing function of its occurrence count among the yet selected phrases list. In practice, the phrases score major contribution is given by events which rarely appears among the yet selected phrases; on the contrary, frequently occurring events give a very small contribution.

\subsection{Score function parameters choice}

The $\alpha_{\mathrm{X}}$ and $\lambda_{\mathrm{X}}$ parameters proper choice allow to achieve the phonological balance among the selected phrases list, once given its size.

The $\lambda_{\mathrm{x}}$ parameters allow to balance the contributions to the phrase score value of the three types of event considered. Equal coverages can be obtained by considering the cardinality of the P, F and $S$ event spaces, i.e. how many different phoneme, SPU and phoneme pairs do exist into the input DB, to be indicated as CP, CF and CS respectively. In order to give more weight to the more populated space, it can be set

$$
\lambda_{x}=\frac{C_{x}}{C_{p}+C_{F}+C_{S}}
$$

The $C_{X}$ values in (4) can be substituted with "subjective" contribution importance opinions.

The $\alpha_{\mathrm{X}}$ magnitudes acts on the score sensitivity with respect to the $\mathrm{x}$-type events count among the selected phrases. A big value gives a very sharp score function. This assure that all the events will be present at least one time, making little difference between different frequency counts. On the contrary, smaller values bring the algorithm to find at least several occurrences for each event; too small dumping values will made the method unable to discriminate among rarely occurred and unseen events, giving an high risk of loosing some event.

A tentative of founding an "optimal" theoretical value for the $\alpha_{X}$ parameters is reported in /4/. It is based on the consideration that, if the algorithm is requested to select $M$ phrases which average phonemic length is $L$, it would be theoretically possible to accommodate $\mu_{\mathrm{X}}=\mathrm{LM} / \mathrm{C}_{\mathrm{X}}$ occurrences of each $\mathrm{x}$-type event. The $\alpha_{\mathrm{X}}$ value can be set to smaller values if there exists enough "space" to accommodate several events occurrences, which is accounted by $\mu_{\mathrm{X}}$. Another speculation suggest to vary the $\alpha_{\mathrm{x}}$ value during the selection iterations, starting with a big value (which forces singletones search) and then decreasing it as the number of phrases grows.

\subsection{Parameter dependency on a priori events importance}

If the user knows to need more occurrences for events to be described with more details, as for recognizers frequent errors, or for particularly relevant speech events for the synthesis quality assessment, adjustments to the score function parameters can be done. In fact, it will be suffice in general to divide the $\alpha_{\mathrm{X}}$ factors by a quantity whose magnitude reflects the relative importance of the event, thus obtaining event-dependent dumping factors. By indicating with $\mathrm{I}_{\mathrm{X}}($. the importance of the general $x$-type event, eq. (2) will become:

$$
S_{x}(\operatorname{Pr})=\frac{1}{N} \sum_{j=1}^{N} e^{-\frac{\alpha_{x} N\left(E_{x}\left(S P U_{j}\right)\right)}{I_{x}\left(E_{x}\left(S P U_{j}\right)\right)}}
$$

\section{SELECTION EXPERIMENTS AND COMPOSITION ANALYSIS}

\subsection{Input Data Base}

The data in input to the algorithm are a set of about 2100 phrases, with a mean length of about 20 SPU for phrase, and a total of about 12.000 words. The DB original composition comes from two main domains. One is a collection of short pieces of text collected form newspapers, novels, romances and textbooks. The other is a technical report about speech recognition. The proposed method has been run to get a total of 60 balanced phrases. 


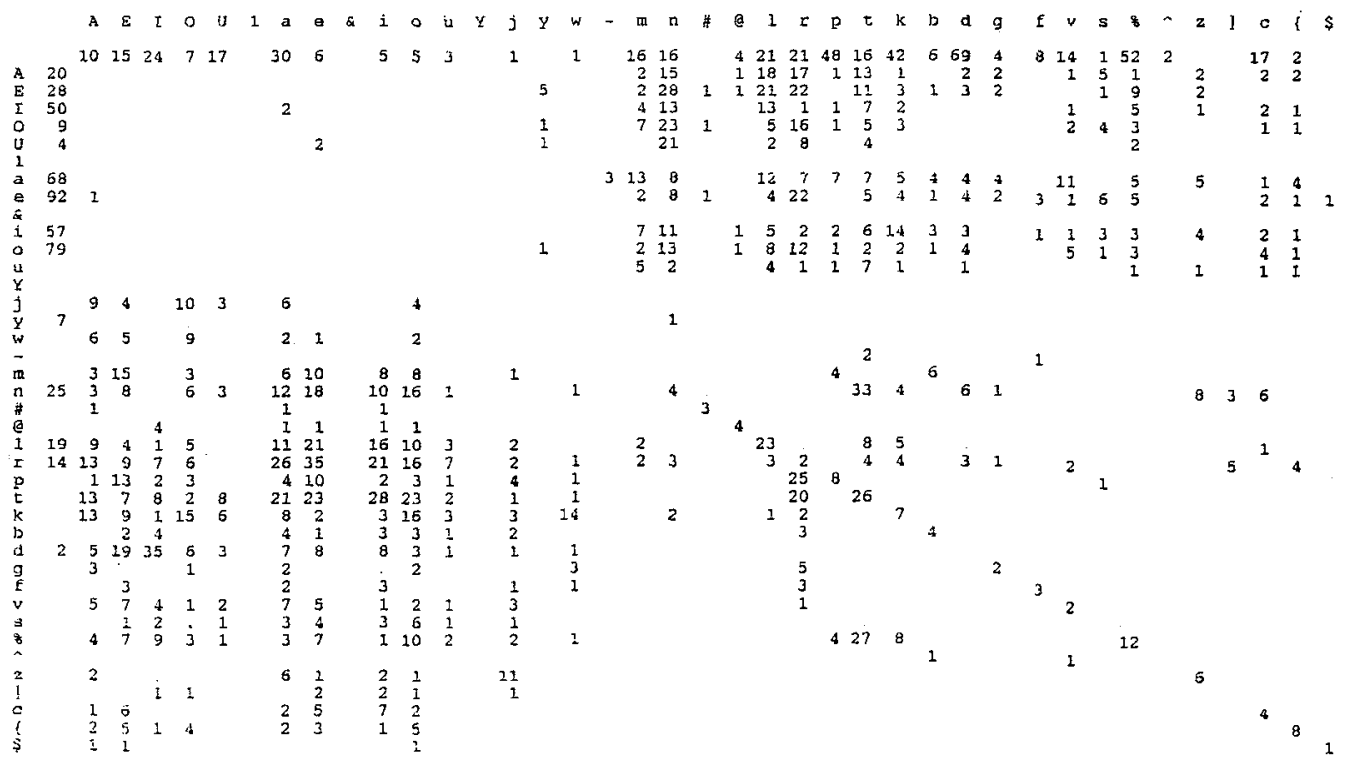

Fig.3 - Phoneme pair ocurrence counts

\subsection{Selected list composition}

Fig. 3 reports the sequential events occurrences count among the 60 selected phrases for the best parameters choice, allowing the collect 465 different phoneme pairs, i.e. about $75 \%$ of the 624 contexts which occur into the input DB. It is important to evidence the twofold properties of the proposed method: occurrences of naturally high frequency events are kept as low as possible, and the frequency of rare events results strongly increased. This behavior is valid also for the other types of events considered, as shown in $/ 4 \%$.

Finally, visual inspection of the results revealed that about $80 \%$ of the selected phrases come from the first part of the input DB. This suggest that much better results can be obtained by utilizing a larger and richer input DB.

\section{CONCLUSIONS AND FUTURE RESEARCH}

Let us remark some features of the proposed method. By first, the utilization of a written text input data base gives meaningful selected phrases, to be naturally read by humans for recognizers training purposes. Second, the specialization of the SPU transcription to the continuous speech /1/ allows to automatically select also inter-word phonological phenomena. Third, the score function parameters can be made dependent on the SPU identity and contexts, allowing to focalize the algorithm attention on events judged to be more relevant for the selected list future use. A natural application of the proposed method is the obtainment of training and test scripts for speech recognizers. Work is in progress for this objective.

Although the selected phrases list yet exhibits an high unpredictability, due to the phrases randomness, collection of the SPU transcription statistics can be compiled in a phonological, statistical model of the language $/ 5 /$, which permits to generate non-existing pseudo words which 'sound' correct from a phonotactical point of view. A long list of nonsense words can be utilized as input DB to the here exposed selection algorithm, in order to obtain a minimum size nonsense words list. Such list could be usefully utilized for text-to-speech synthesizers intelligibility assessment test.

\section{REFERENCES}

1// - A. Falaschi, Proc of Eurospeech 89, th ECST, 26-29 Sept. 1989, Paris

12/ - A.Falaschi, PhD Thesis, La Sapienza Univ. of Rome, INFOCOM Dpt., Feb. 1989

13) - L.F.Lamel, R.H.Kassel, S.Seneff, Proc. DARPA Speech Recognition Workshop, Palo Alto, CA, Feb 86; also in Proc. of the ESCA Workshop on speech 1/O assessment and data bases, Noordwjikerout, The Netherlands, Sept. 89

14/ - A.Falaschi, Proc. of the ESCA Workshop on speech I/O assessment and data bases, Noordwjikerout, The Netherlands, Sept. 89

15/ - A. Falaschi, 1st European Conference on Speech Technology, Sept 87, Edinburgh -UK 